\title{
Mercurial-resistance determinants in Pseudomonas strain K-62 plasmid pMR68
}

\author{
Yuka Sone', Yusuke Mochizuki ${ }^{1}$, Keita Koizawa', Ryosuke Nakamura' ${ }^{1}$, Hidemitsu Pan-Hou' ${ }^{2}$, Tomoo Itoh \\ and Masako Kiyono ${ }^{1 *}$
}

\begin{abstract}
We report the complete nucleotide sequence of plasmid pMR68, isolated from Pseudomonas strain K-62, two plasmids contribute to broad-spectrum mercury resistance and that the mer operon from one of them (pMR26) has been previously characterized. The plasmid was 71,020 bp in length and contained 75 coding regions. Three mer gene clusters were identified. The first comprised merR-orf4-orf5-merT1-merP1-merF-merA-merB1, which confers bacterial resistance to mercuric ions and organomercury. The second and third clusters comprised merT2-merP2, which encodes a mercury transport system, and merB2, which encodes an organomercurial lyase, respectively. The deduced amino acid sequences for the proteins encoded by each of the mer genes identified in pMR68 bore greater similarity to sequences from Methylobacterium extorquens AM1 than to those from pMR26, a second mercury-resistance plasmid from Pseudomonas strain K-62. Escherichia coli cells carrying pMKY12 (containing merR-orf4-orf5-merT1-merP1-merF-merA-merB1 cloned from pMR68) and cells carrying pMRA114 (containing merR-merT-merP-merA-merG-merB1 cloned from plasmid pMR26) were more resistant to, and volatilized more, mercury from mercuric ions and phenylmercury than the control cells. The present results, together with our earlier findings, indicate that the high phenylmercury resistance noted for Pseudomonas strain K-62 seems to be achieved by multiple genes, particularly by the multiple merB encoding organomercurial lyase and one merG encoding cellular permeability to phenylmercury. The novel mer gene identified in pMR68 may help us to design new strategies aimed at the bioremediation of mercurials.
\end{abstract}

Keywords: Mercury resistance; mer operon; Plasmid pMR68; Pseudomonas strain K-62

\section{Introduction}

Pseudomonas strain K-62, a bacterial strain isolated from phenylmercury-polluted soil, is about 1,000 times more resistant to phenylmercury than sensitive strains of Escherichia coli (Tonomura et al. 1968). A study performed about 40 years ago showed that the biochemical mechanism underlying this mercurial resistance is based on the enzymatic degradation of organomercurials and the subsequent reduction of the resulting mercuric ions to the less toxic and more volatile metallic mercury (Tonomura et al. 1968). Two separate organomercurial lyases, designated S-1 and S-2, each showing somewhat different physical properties and substrate specificities, are thought to be responsible for the resistance of $P$. K-62 to phenylmercury (Tezuka and Tonomura 1976;

\footnotetext{
* Correspondence: kiyonom@pharm.kitasato-u.ac.jp

${ }^{1}$ Department of Public Health and Molecular Toxicology, School of Pharmacy, Kitasato University, 5-9-1 Shirokane, Tokyo, Minato-ku 108-8641, Japan Full list of author information is available at the end of the article
}

Tezuka and Tonomura 1978). The organomercurial resistance of this soil strain is encoded by two plasmids, pMR26 and pMR68 (Kiyono et al. 1995b). In addition, pMR26 contains two mer operons that map about $1 \mathrm{~kb}$ apart (Kiyono et al. 1997). One comprises merR-o/pmerT-merP-merA-merG-merB1. The other is a defective mer operon comprising merR-o/p-merB2-merD (Kiyono and Pan-Hou 1999a).

Studies suggest that merR is a regulatory gene that both negatively and positively controls the transcription of merTPABD (Ansari et al. 1995; Brown et al. 2003), whereas $\mathrm{MerD}$ is a transcriptional co-regulator (Hobman and Brown 1997; Kiyono et al. 1995a; Lund and Brown 1987). MerT, merP, merA, and merB encode a membrane $\mathrm{Hg}^{2+}$-transport protein (Hobman and Brown 1997; Kiyono et al. 1995a; Lund and Brown 1987), a periplasmic $\mathrm{Hg}^{2+}$-binding protein (Hobman and Brown 1997; Kiyono et al. 1995a; Kiyono et al. 2000; Lund and Brown 1987), a mercuric reductase (Hobman and Brown 1997; Schiering 
et al. 1991; Silver and Phung 1996), and a organomercurial lyase (Griffin et al. 1987; Lafrance-Vanasse et al. 2009; Miller 1999), respectively. $\operatorname{mer} G$, identified in pMR26, is a newly-identified mer-gene involved in phenylmercury resistance, which is thought to act by reducing cell permeability to phenylmercury (Kiyono and Pan-Hou 1999b). Taken together, these findings suggest that the high resistance to phenylmercury shown by $P$. K-62 is mediated by the two functional organomercurial lyase enzymes encoded by pMR26 merB1 and merB2 (Kiyono et al. 1995b; Kiyono and Pan-Hou 1999a), by changes in cellular permeability to phenylmercury mediated by merG (Kiyono and Pan-Hou 1999b), and by an presumptive mer operon located on plasmid pMR68 (Kiyono et al. 1995b) However, no mercurial-resistance loci have been identified in pMR68; indeed, a previous study shows that removal of pMR26 from strain K-62 does not alter its mercurial-resistant phenotype nor prevent it from volatilizing $\mathrm{Hg}^{2+}$ and organomercurials (Kiyono et al. 1995b).

To fully explain the high resistance to phenylmercury observed in this strain of soil bacteria, it is essential to understand the mer genes expressed by pMR68. The aim of the present study was to completely identify the mercury resistance genes of plasmid pMR68 isolated from strain K-62. The mer genes encoded by pMR68 were then cloned and analyzed.

\section{Materials and methods}

\section{Bacterial strains and culture conditions}

$P$. strain K-62, isolated from phenylmercury-polluted soil in Japan (Tonomura et al. 1968) and deposited in a culture collection belonging to the WDCM56, was kindly supplied by Dr. K. Tonomura and grown in nutrient broth as previously described (Tezuka and Tonomura 1976) (see Table 1). E. coli XL1-Blue was grown at $37^{\circ} \mathrm{C}$ in Luria-Bertani (LB) medium. Antibiotics or mercuric chloride were added to the medium at the following concentrations when appropriate: ampicillin, $100 \mu \mathrm{g} / \mathrm{ml}$ (E. coli); mercuric chloride, $40 \mu \mathrm{g} / \mathrm{ml}$.

\section{DNA purification}

Six plasmids (8.5-82 kb) (Kiyono et al. 1995b) were purified from strain K-62 grown in nutrient broth for 3 days at $30^{\circ} \mathrm{C}$ according to the method of Sasakawa et al. (Sasakawa et al. 1986). The purified plasmids were loaded into the wells of a $0.7 \%$ low-melting temperature agarose gel (SeaPlaque GTG agarose, Lonza Rockland, Inc., Rockland, ME) and electrophoresed in $0.5 \times \mathrm{TBE}(45 \mathrm{mM}$ Tris $\mathrm{HCl}, 45 \mathrm{mM}$ boric acid, $1 \mathrm{mM}$ EDTA) in a contour clamped homogeneous electric field (CHEF) DRII device (Bio-Rad Laboratories, Hercules, CA) with the pulse-time ramped from 10 to $60 \mathrm{~s}(6 \mathrm{~V} / \mathrm{cm})$ for $16 \mathrm{~h}$ at $14^{\circ} \mathrm{C}$. The gels were then stained with ethidium bromide. The band corresponding to the pMR68 plasmid was excised and equili-

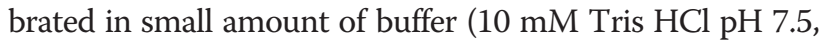
$0.25 \mathrm{mM}$ EDTA, $100 \mathrm{mM} \mathrm{NaCl})$. The agarose was melted at $68^{\circ} \mathrm{C}$ and digested with $\beta$-agarase (New England Biolabs, Hertfordshire, England). The pMR68 plasmid was then concentrated by ethanol precipitation.

\section{DNA sequencing}

Purified pMR68 plasmid DNA $(15 \mu \mathrm{g})$ was sequenced by Eurofins MWG Operon (Ebersberg, Germany) using a Genome Sequencer FLX Titanium system (Roche, Basel, Switzerland). Shotgun sequencing was then performed on the range of one region of a 16-region picotiter plate, resulting in 33,964 reads with an average length of $340 \mathrm{bp}$. The sequences were assembled using Celera Assembler Version 5.3, generating 18 contigs of at least $1,000 \mathrm{bp}$ in length, some of which were high coverage ( $>100$-fold). Connections between these contigs and adjacent contigs with similarly high coverage were identified by looking for

Table 1 Strains and plasmids used in this study

\begin{tabular}{|c|c|c|c|}
\hline Stains and plasmids & \multicolumn{2}{|l|}{ Description or relevant feature(s) } & Reference or source \\
\hline \multicolumn{4}{|l|}{ Strains } \\
\hline E. coli XL1-Blue & \multicolumn{2}{|c|}{ recA1 endA1 gyrA96 thi hsdR17 supE44 relA1 lac/ [F':.Tn 10 proAB+laclq lacZM15 traD36] } & Bullock et al. (1987) \\
\hline P. strain K-62 (wild) & $82,68,56,31,26,8.5 \mathrm{~kb}$ plasmids & IC50 of mercuric chloride; 100 ppm, mercury vapor activity; + & Kiyono et al. (1995b) \\
\hline P. strain K-62 (mutant 26) & $82,68,56,31,8.5 \mathrm{~kb}$ plasmids & IC50 of mercuric chloride; 50 ppm, mercury vapor activity; + & Kiyono et al. (1995b) \\
\hline P. strain K-62 (mutant 68) & $82,56,31,26,8.5 \mathrm{~kb}$ plasmids & IC50 of mercuric chloride; 17 ppm, mercury vapor activity; + & Kiyono et al. (1995b) \\
\hline P. strain K-62 (mutant TY) & $82,56,31,8.5 \mathrm{~kb}$ plasmids & IC50 of mercuric chloride; 2 ppm, mercury vapor activity; - & Kiyono et al. (1995b) \\
\hline \multicolumn{4}{|l|}{ Plasmids } \\
\hline pMR26 & \multicolumn{2}{|l|}{26 kb plasmids from $P$. strain $\mathrm{K}-62$} & Kiyono et al. (1997) \\
\hline pMR68 & \multicolumn{2}{|l|}{$68 \mathrm{~kb}$ plasmids from $P$. strain $\mathrm{K}-62$} & This study \\
\hline pUC118 & \multicolumn{2}{|l|}{ None; cloning vector } & Vieira et al. (1987) \\
\hline pMRA17 & \multicolumn{2}{|c|}{ merR-o/p-merT-merP-merA-merG-merB1 of pMR26 in pBluescriptll } & Kiyono et al. (1997) \\
\hline PMRA114 & \multicolumn{2}{|c|}{ merR-o/p-merT-merP-merA-merG-merB1 of pMR26 in pUC118 } & This study \\
\hline pMKY12 & \multicolumn{2}{|c|}{ merR-o/p-orf4-orf5-merT1-merP1-merA-merB1 of pMR68 in pUC118 } & This study \\
\hline
\end{tabular}


sequencing reads that were split between contigs by the assembly software. Using this process, a chain of seven contigs was assembled into a $71,020 \mathrm{bp}$ sequence. The joins between the seven contigs were checked by PCR using the following primer pairs: $1 \mathrm{U}-68 \mathrm{~kb}-15850$ and $8 \mathrm{~L}-$ 68 kb-16600; 2U-68 kb-18520 and 9 L-68 kb-19216; 3U-68 kb-19421 and 10 L-68 kb-20259; 4U-68 kb-38451 and 11 L-68 kb-39477; 5U-68 kb-42220 and 12 L-68 kb43396; 6U-68 kb-67929 and 13 L-68 kb-68480; and 7U$68 \mathrm{~kb}-70484$ and 14 L-68 kb-457 (see Additional file 1: Table S1).

After the complete nucleotide sequence of pMR68 was obtained, potential open reading frames (ORFs) was searched using the program of genetic information processing software (Genetyx corporation, Tokyo, Japan) and using protein BLAST (http://blast.ncbi.nlm.nih.gov/ Blast.cgi) to confirm the results. Conserved domains were identified searching for Clusters of Orthologous Groups of proteins (COGs) in the NCBI data base. The molecular weights of the encoded proteins were determined by ProtParam (Swiss Institute of Bioinformatics; http://www.expasy.ch/tools/protparam.html). The annotated sequence of pMR68 was deposited in the NCBI database under Accession No. NC019309.

\section{Gene cloning and analysis of the mer operon}

Plasmid pMKY12 was constructed as follows: Plasmid pMR68 (accession no. NC019309) was used as the template for PCR amplification (PrimeSTAR GXL DNA polymerase, Takara Bio, Inc., Otsu, Japan) of a $10.3 \mathrm{~kb}$ fragment containing the merR-o/p-ofr4-orf5-merT1merP1-merF-merA-merB1 genes. The primers used were 16U-68 kb-2393 and 21 L-68 kb-9566 (Additional file 1: Table S1). After blunting and 5'-phosphorylation of the DNA fragment using a Mighty Cloning Kit (Blunt End) (Takara Bio, Inc., Otsu, Japan), the DNA fragment was cloned into the blunt-ended (Hinc II) vector, pUC118 (Vieira and Messing 1987).

Plasmid pMRA114 was constructed as follows: Plasmid pMRA17, containing a $6.6 \mathrm{~kb}$ merR-o/p-merT-merPmerA-merG-merB1 fragment from pMR26 (accession no. D83080), which contains restriction sites for SacI, was used as the starting material. After digestion with SacI, the $6.6 \mathrm{~kb}$ fragment was cloned into the corresponding sites in pUC118. The integrity of all cloned fragments was confirmed by sequencing.

\section{Mercury susceptibility tests and volatilization activity tests}

The resistance of E. coli XL1-Blue carrying pUC118 (control vector), pMRA114, or pMKY12 to $\mathrm{HgCl}_{2}$ or $\mathrm{C}_{6} \mathrm{H}_{5} \mathrm{HgOCOCH}_{3}$ was determined in liquid medium. E. coli cells carrying the control or recombinant plasmids were grown overnight in $\mathrm{LB}$ medium at $37^{\circ} \mathrm{C}$. Cells were harvested and suspended in LB medium $\left(1.6 \times 10^{7}\right.$ cells/
$200 \mu \mathrm{L} /$ well) containing $\mathrm{HgCl}_{2}$ or $\mathrm{C}_{6} \mathrm{H}_{5} \mathrm{HgOCOCH}_{3}$ at various concentrations. After incubation at $37^{\circ} \mathrm{C}$ for $16 \mathrm{~h}$, the absorbance of each culture was read at $\mathrm{A}_{600}$ to measure cell growth.

The mercury volatilization assay was performed as follows: E. coli cells carrying the control or recombinant plasmids were grown to mid-exponential phase and then suspended in $\mathrm{LB}$ medium containing $50 \mu \mathrm{M} \mathrm{HgCl}_{2}$ or $5 \mu \mathrm{M} \mathrm{C} \mathrm{C}_{5} \mathrm{H}_{5} \mathrm{HOCOCH}_{3}$. After incubating at $37^{\circ} \mathrm{C}$ for $16 \mathrm{~h}$, the samples were digested with concentrated nitric acid for $2 \mathrm{~h}$ at $90^{\circ} \mathrm{C}$ and the amount of mercury remaining in the medium was determined by flameless cold-vapour atomic adsorption spectrometry using an atomic mercury analyser (HG-310; Hiranuma, Japan).

\section{Results}

\section{General features of plasmid pMR68}

The complete nucleotide sequence of plasmid pMR68 was assembled into a circular DNA sequence comprising $71,020 \mathrm{bp}$, with an overall $\mathrm{G}+\mathrm{C}$ content of $64.5 \%$. The sequence showed that pMR68 was $3 \mathrm{~kb}$ larger than previously calculated on the basis of agarose gel electrophoresis (Kiyono et al. 1995b). Figure 1 shows a detailed genetic map of pMR68. The predicted coding regions showed a particular genetic organization, highlighting two well-defined regions that corresponded to genomic islands (comprising $41 \mathrm{~kb}$ of the complete plasmid). The first region possessed a $21 \mathrm{~kb}$ island (pMR68 co-coordinates: $59,285-71,020$ and $1-9,472$ ), which contained genes involved in mercurial resistance and mobility (Figure 1). The second region possessed a $20 \mathrm{~kb}$ island (pMR68 co-coordinates; 10,189-30,567), which contained plasmid transposable elements (Figure 1). A summary of the sequence data for pMR68, including the length and molecular mass of the predicted proteins and their sequence homology with known proteins, is shown in Additional file 2: Table S2.

\section{Mercury-resistance $\left(\mathrm{Hg}^{\mathrm{R}}\right)$ determinants}

The pMR68 plasmid contained three putative mercuryresistance (mer) gene clusters (Figure 1): Cluster 1) a potential mer operon, consisting of the merR-orf4-orf5T1-P1-F-A-B1 genes (ORFs 3 and 6-10); Cluster 2) mercury transport genes, merT2-P2 (ORFs 62 and 63); and Cluster 3) an organomercurial lyase gene, merB2 (ORF 72) (Additional file 2: Table S2 and Figures 1 and 2). The potential mer operon within pMR68 was located between putative transposable elements, and flanked by genes encoding a transposase IS4 family protein (ORF 75) and a transposase IS5 family protein (ORF 11) (Additional file 2: Table S2). The incomplete mer operon (cluster 2) within pMR68 was also located between putative transposable elements, and flanked by genes encoding a transposase IS30 family protein (ORF 56) and a transposase IS116 family 


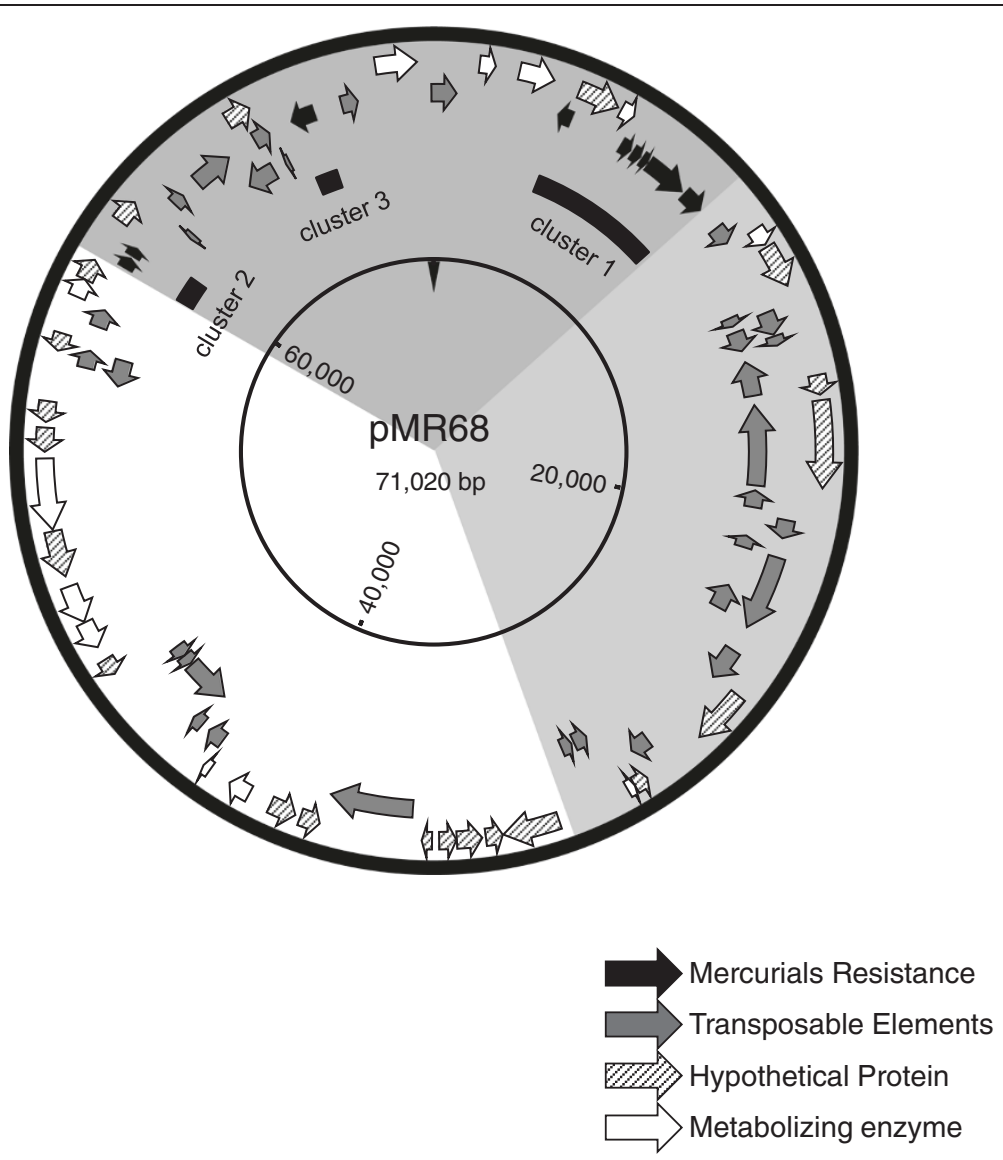

Figure 1 Genetic map of the $P$. strain K-62 plasmid, pMR68. The deduced coding regions are shown by open arrows indicating the direction of transcription. The circular positions are indicated at intervals of 20,000 bp. The gene clusters responsible for mercurial resistance, transposable elements, metabolizing enzymes, and hypothetical proteins are indicated by the black, gray, open and shaded arrows, respectively.

protein (ORF 68) (Additional file 2: Table S2). The DNA sequence showed the presence of putative promoters upstream of the merT1 genes within the complete mer operon of cluster 1 (Figure 2). Upstream of merR and upstream of $m e r T 1$ were sequences containing potential -35 (ATCAGA) and -10 (GATTAT) and -35 (TTGCAC) and -10 (CATAAT) sequences, and a dyad symmetrical sequence (GCACCTGTAGCCGCTACAGGTTG), respectively, which could be interpreted as an operator/promoter (o/p) sequence (cluster 1, Figure 2).

ORF4 (encoding a hypothetical protein (HP)) and ORF5 (showing 63\% identity to phosphoribosyl-AMP cyclohydrolase from Parvibaculum lavamentivorans DS-1) were located between MerR and MerT1. Two sets of merT, merP, and merB genes were found in pMR68, and the similarities between merT1 and merT2, merP1 and merP2, and merB1 and merB2 ranged from $27 \%$ to $69 \%$ at both the nucleic acid and amino acid levels (Figure 2). The sequence similarity between the pMR68 and pMR26 proteins (Kiyono et al. 1997; Kiyono and Pan-Hou 1999a) from strain K-62 or the well-known pDU1358 (Griffin et al. 1987) and Tn21 (Gilbert and Summers 1988; Liebert et al.
1999) proteins from Gram-negative bacteria was low (Table 2).

\section{Gene cloning and analysis of the mer operon}

To identify the physiological role played by the mer operons in pMR68 and pMR26 in E. coli, we constructed recombinant plasmids pMKY12 and pMRA114, which contained the merR-orf4-orf5-merT1-merP1-merFmerA-merB1 genes from pMR68 and the merR-merTmerP-merA-merG-merB1 genes from pMR26, respectively (Table 1). Bacteria containing pMKY12 showed greater resistance to $\mathrm{Hg}$ (II) than control cells carrying plasmid pUC118; the level of resistance was almost the same as that shown by cells containing pMRA114 (Figure 3A).

We next examined the volatilization of mercury from $\mathrm{Hg}$ (II) and $\mathrm{C}_{6} \mathrm{H}_{5} \mathrm{Hg}$ (I) by cells containing a control vector (pUC118) or the pMKY12 or pMRA114 plasmids. As shown in Figure 4, cells carrying both pMKY12 and pMRA114 were able to volatilize mercury from both $\mathrm{Hg}(\mathrm{II})$ and $\mathrm{C}_{6} \mathrm{H}_{5} \mathrm{Hg}$ (I). Volatilization of $\mathrm{Hg}(\mathrm{II})$ was similar between cells carrying pMKY12 or pMRA114; however, volatilization of $\mathrm{C}_{6} \mathrm{H}_{5} \mathrm{Hg}(\mathrm{I})$ was significantly higher in cells 


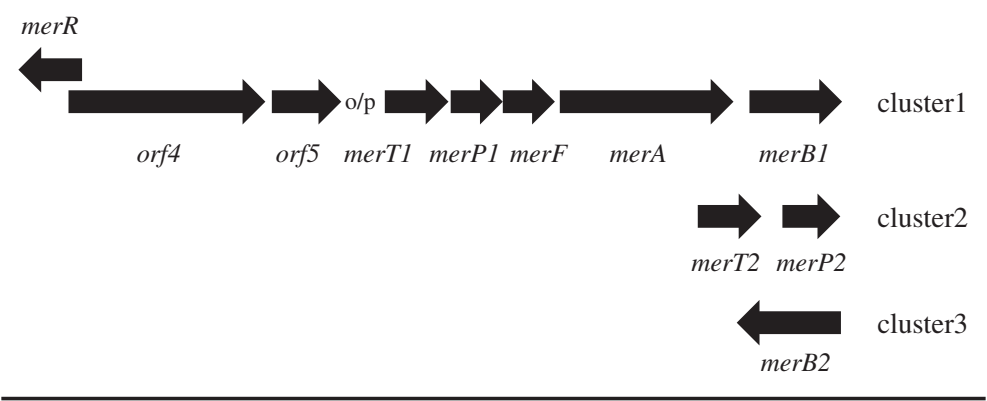

\begin{tabular}{|c|c|c|c|c|}
\hline \multicolumn{5}{|c|}{ Comparison of nucleotide and amino acid sequence homology } \\
\hline mer genes & & merT1: $\operatorname{merT2}$ & merPI: $\operatorname{merP2}$ & merB1: merB2 \\
\hline \multirow{2}{*}{$\begin{array}{c}\text { homology } \\
(\%)\end{array}$} & nucleotide & 67.9 & 69.0 & 50.3 \\
\hline & amino acid & 64.4 & 63.4 & 27.2 \\
\hline
\end{tabular}

Figure 2 Organization of the mer operon of pMR68 and its homology with the corresponding mer genes identified in pMR68. merR, regulatory gene; merT and merF, mercury transport genes; merP, mercury binding gene; merA, mercuric reductase gene; mer $B$, organomercurial lyase gene; orf, unknown open reading frame.

carrying pMKY12 than in those carrying pMRA114 (Figure 4B). A volatilization test indicated that the cells carrying pMKY12 were able to volatilize almost $90 \%$ of the total phenylmercury.

\section{Discussion}

The present study determined the complete nucleotide sequence of plasmid pMR68 (isolated from $P$. strain K-62). In addition, the mer genes in pMR68 and pMR26 were identified, sequenced, and cloned in E. coli. The pMR68 sequence contained 75 complete coding regions; however, we were not able to identify a predicted origin of replication (Additional file 2: Table S2 and Figure 1). Although most of the identified genes (44\%) encoded mobile elements related to transfer functions, $12 \%$ encoded mercurial-resistance determinants, $16 \%$ encoded metabolism-related genes, and $28 \%$ encoded hypothetical proteins (HPs).

One of the three mer-gene clusters in pMR68 (merRorf4-orf5-merT1-merP1-merF-merA-merB1) was identified as a potential mer operon, which confers bacterial resistance to both inorganic and organic mercury (Additional file 2: Table S2 and Figure 3). The number and order of the mer genes on this potential mer operon were different to those of merR1-o/p-T-P-A-G-B1 of pMR26 (which encodes resistance to both inorganic and organic mercury) and merR2-o/p-B2-D of pMR26 (which confers bacterial hypersensitivity to organomercury compounds) (Table 2 and Figures 1 and 2) (Kiyono et al. 1997; Kiyono and Pan-Hou 1999a; Kiyono et al. 1997). The deduced amino acid sequences for the proteins encoded by the mer genes in pMR68 were more similar (67-84\%) to those of a putative mer operon in Methylobacterium extorquens AM1, which lacks the merB gene (Vuilleumier et al. 2009), than to those in pMR26 (21-47\%) (Table 2).

We identified potential -35 and -10 sequences and a dyad symmetrical sequence lying upstream of pMR68 merR and upstream of merT1, respectively; these sequences may represent putative promoters of the mer

Table 2 Amino acid sequence homology of mer genes between pMR68 and pMR26, pDU1358, Tn21 or M. extorquens AM1 putative mer operon

\begin{tabular}{|c|c|c|c|c|c|c|c|c|c|}
\hline \multicolumn{10}{|c|}{$\%$ of amino acid sequence homology } \\
\hline pMR68 & MerR & MerT1 & MerT2 & MerP1 & MerP2 & MerF & MerA & MerB1 & MerB2 \\
\hline pMR26 & 32.4 & 38.8 & 47.0 & 31.3 & 36.0 & - & 35.4 & 21.3 & 22.0 \\
\hline pDU1358 & 32.5 & 38.8 & 45.5 & 31.2 & 36.0 & - & 26.7 & 21.0 & 19.7 \\
\hline $\operatorname{Tn} 21$ & 31.8 & 38.8 & 47.0 & 31.1 & 38.7 & - & 38.6 & - & - \\
\hline M. extorquens AM1 & 73.0 & 76.0 & 71.0 & 76.0 & 67.0 & - & 84.0 & - & - \\
\hline
\end{tabular}




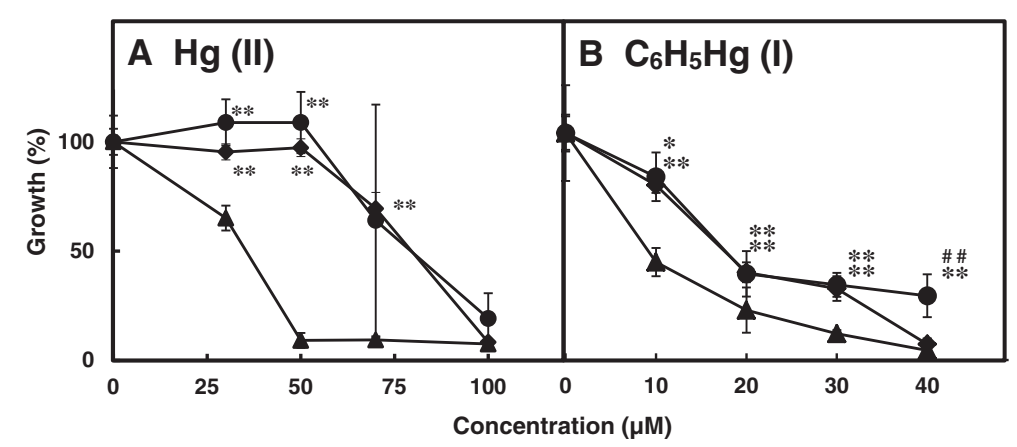

Figure 3 Susceptibility to mercurials. E. coli cells carrying pUC118 (filled triangles), pMKY12 (filled circles), or pMRA114 (filled diamonds) were grown in liquid medium containing varying concentrations of $\mathrm{HgCl}_{2}(\mathbf{A})$ or $\mathrm{C}_{6} \mathrm{H}_{5} \mathrm{HgOCOCH}_{3}(\mathbf{B})$. Growth was estimated by measuring the turbidity at $600 \mathrm{~nm}$. Data represent the mean \pm S.D. of triplicate measurements from three independent experiments. ${ }^{*} p<0.05 \mathrm{vs}$. control. ${ }^{* *} p<0.01$ vs. control. ${ }^{\# \#} p<0.01$ vs. pMRA114.

operon of cluster 1 in pMR68. The distance between the -35 and -10 positions within the putative pMR68 merR promoter was $19 \mathrm{bp}$. The spacing between the merR promoter and Tn21 in pMR26 was also $19 \mathrm{bp}$, which is essential for the "twist and bend" mechanism underlying transcriptional activation (Ansari et al. 1995; Kiyono et al. 1997; Kiyono and Pan-Hou 1999a). Taken together, these observations suggest that merT1-P1-F-A-B1 is regulated by both the merR gene and the mer operator.

Volatilization of mercury from organomercurials is thought to result from the degradation of organic mercury by the organomercurial lyase encoded by $\operatorname{mer} B$, followed by the reduction of the resulting $\mathrm{Hg}^{2+}$ to volatile $\mathrm{Hg}^{0}$ by the mercuric reductase encoded by merA (Barkay et al. 2003; Silver and Phung le 2005). Cells carrying pMKY12 (containing merR-orf4-orf5-merT1-merP1-merFmerA-merB1 cloned from pMR68) and cells carrying
pMRA114 (containing merR-merT-merP-merA-merGmerB1 cloned from plasmid pMR26) were more resistant to, and volatilized more, mercury from mercuric chloride and phenylmercuric acetate than the control cells (Figures 3 and 4).

The present results, together with those of our previous study, suggest that the high resistance to phenylmercury shown by strain K-62 may be due to the following: [1] the functional organomercurial lyase enzymes encoded by pMR26 merB1 and merB2 (Kiyono et al. 1995b; Kiyono et al. 1997; Kiyono and Pan-Hou 1999a), and pMR68 merB1; [2] the two functional mercuric reductases encoded by pMR26 merA (Kiyono et al. 1995b; Kiyono and PanHou 1999a) and pMR68 merA; [3] the multi-functional transporters encoded by pMR26 merT and merP (Kiyono et al. 1995a; Kiyono et al. 2000; Nagata et al. 2006; Uno et al. 1997), and by pMR68 merT1-merP1-merF; and [4]

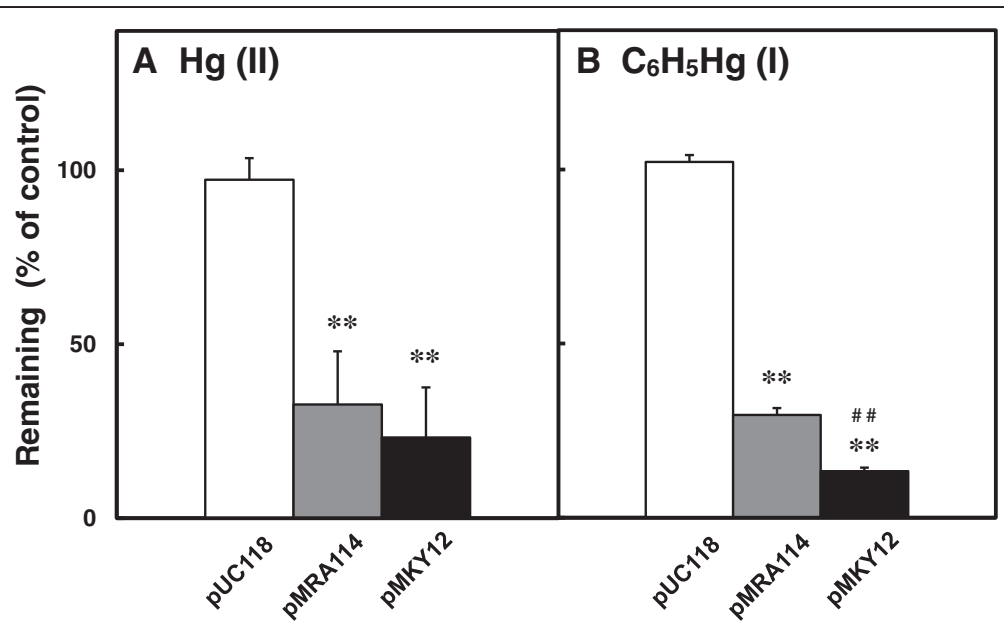

Figure 4 Volatilization of mercury from $\mathbf{H g}(\mathrm{II})$ and $\mathrm{C}_{6} \mathrm{H}_{5} \mathrm{Hg}(\mathrm{I})$. E. coli cells carrying pUC118 (empty bar), pMRA114 (shaded bar), or pMKY12 (black bar) were grown in liquid medium containing $50 \mu \mathrm{M} \mathrm{HgCl}_{2}$ (A) or $5 \mu \mathrm{M} \mathrm{C}_{6} \mathrm{H}_{5} \mathrm{HgOCOCH}_{3}$ (B). After incubation at $37^{\circ} \mathrm{C}$ for $16 \mathrm{~h}$, the amount of mercury remaining in the medium was measured as described in "Materials and Methods". Data represent the mean \pm S.D. of triplicate measurements from three independent experiments. ${ }^{* *} p<0.01$ vs. control. ${ }^{\# \#} p<0.01$ vs. pMRA114. 
alterations in cellular permeability to phenylmercury mediated by pMR26 merG (Kiyono and Pan-Hou 1999b). Further analysis of orf4 and orf5 within pMR68 is currently on-going.

In conclusion, sequence analysis of pMR68 showed that the plasmid contains novel genes that may provide Pseudomonas strains with the means to adapt to a wide variety of challenging environments, including exposure to heavy metals. Such resistance mechanisms are likely to be linked to the evolution of the bacterial hosts. The novel mer gene identified in pMR68 may help us to design new strategies aimed at the bioremediation of mercurycontaining compounds present in the environment.

\section{Additional files}

Additional file 1: Table S1. Oligonucleotide primers used in this study. Additional file 2: Table S2. Summary of location of predicted coding regions on plasmid pMR68.

\section{Competing interests}

The authors declare that they have no competing interests.

\section{Acknowledgments}

We thank Miss M. Suzuki, Mr. M. Saito and Mr. I. Okuno for their technical assistance. This work was supported in part by a Grant-in-Aid for Young Scientists (B) (No. 24790128) to Y.S. and a Grant-in-Aid for Scientific Research (C) (No. 24510104) to M.K. from the Ministry of Education, Science and Culture, Japan.

\section{Author details}

'Department of Public Health and Molecular Toxicology, School of Pharmacy, Kitasato University, 5-9-1 Shirokane, Tokyo, Minato-ku 108-8641, Japan.

${ }^{2}$ Faculty of Pharmaceutical Sciences, Setsunan University, 45-1

Nagaotoge-cho, Osaka, Hirakata 573-0101, Japan.

Received: 5 July 2013 Accepted: 24 July 2013

Published: 28 July 2013

\section{References}

Ansari AZ, Bradner JE, O'Halloran TV (1995) DNA-bend modulation in a repressorto-activator switching mechanism. Nature 374(6520):371-375. doi:10.1038/ 374370a0.

Barkay T, Miller SM, Summers AO (2003) Bacterial mercury resistance from atoms to ecosystems. FEMS Microbiol Rev 27(2-3):355-384. doi:10.1016/s0168-6445 (03)00046-9.

Brown NL, Stoyanov JV, Kidd SP, Hobman JL (2003) The MerR family of transcriptional regulators. FEMS Microbiol Rev 27(2-3):145-163

Bullock WO, Fernandez JM, Short JM (1987) XL1-Blue: A high efficiency plasmid transforming recA Escherichia coli strain with beta-galactosidase selection. Biotechniques 5(4):376-379

Gilbert MP, Summers AO (1988) The distribution and divergence of DNA sequences related to the Tn21 and Tn501 mer operons. Plasmid 20(2):127-136

Griffin HG, Foster TJ, Silver S, Misra TK (1987) Cloning and DNA sequence of the mercuric- and organomercurial-resistance determinants of plasmid pDU1358. Proc Natl Acad Sci USA 84(10):3112-3116

Hobman JL, Brown NL (1997) bacterial mercury-resistance genes. Met lons Biol Syst 34:527-568

Kiyono M, Pan-Hou H (1999a) DNA sequence and expression of a defective mer operon from Pseudomonas K-62 plasmid pMR26. Biol Pharm Bull 22(9):910-914

Kiyono M, Pan-Hou H (1999b) The merG gene product is involved in phenylmercury resistance in Pseudomonas strain K-62.J Bacteriol 181(3):726-730

Kiyono M, Omura T, Fujimori H, Pan-Hou H (1995a) Lack of involvement of merT and merP in methylmercury transport in mercury resistant Pseudomonas K-62. FEMS Microbiol Lett 128(3):301-306
Kiyono M, Omura T, Fujimori H, Pan-Hou H (1995b) Organomercurial resistance determinants in Pseudomonas K-62 are present on two plasmids. Arch Microbiol 163(4):242-247

Kiyono M, Omura T, Inuzuka M, Fujimori H, Pan-Hou H (1997) Nucleotide sequence and expression of the organomercurial-resistance determinants from a Pseudomonas K-62 plasmid pMR26. Gene 189(2):151-157

Kiyono M, Uno Y, Omura T, Pan-Hou H (2000) Role of MerT and MerP from Pseudomonas K-62 plasmid pMR26 in the transport of phenylmercury. Biol Pharm Bull 23(3):279-282

Lafrance-Vanasse J, Lefebvre M, Di Lello P, Sygusch J, Omichinski JG (2009) Crystal structures of the organomercurial lyase MerB in its free and mercurybound forms: insights into the mechanism of methylmercury degradation. J Biol Chem 284(2):938-944. doi:10.1074/jbc.M807143200.

Liebert CA, Hall RM, Summers AO (1999) Transposon Tn21, flagship of the floating genome. Microbiol Mol Biol Rev 63(3):507-522

Lund PA, Brown NL (1987) Role of the merT and merP gene products of transposon $\mathrm{Tn} 501$ in the induction and expression of resistance to mercuric ions. Gene 52(2-3):207-214

Miller SM (1999) Bacterial detoxification of $\mathrm{Hg}(\mathrm{II})$ and organomercurials Essays Biochem 34:17-30

Nagata T, Kiyono M, Pan-Hou H (2006) Involvement of aromatic amino acids in phenylmercury trasport by MerT protein. J Health Sci 52(4):475-477

Sasakawa C, Kamata K, Sakai T, Murayama SY, Makino S, Yoshikawa M (1986) Molecular alteration of the 140-megadalton plasmid associated with loss of virulence and Congo red binding activity in Shigella flexneri. Infect Immun 51(2):470-475

Schiering N, Kabsch W, Moore MJ, Distefano MD, Walsh CT, Pai EF (1991) Structure of the detoxification catalyst mercuric ion reductase from Bacillus sp. strain RC607. Nature 352(6331):168-172. doi:10.1038/352168a0.

Silver S, le Phung T (2005) A bacterial view of the periodic table: genes and proteins for toxic inorganic ions. J Ind Microbiol Biotechnol 32(11-12):587-605. doi:10.1007/s10295-005-0019-6.

Silver S, Phung LT (1996) Bacterial heavy metal resistance: new surprises. Annu Rev Microbiol 50:753-789. doi:10.1146/annurev.micro.50.1.753.

Tezuka T, Tonomura K (1976) Purification and properties of an enzyme catalyzing the splitting of carbon-mercury linkages from mercury-resistant Pseudomonas K-62 strain. I. Splitting enzyme 1. J Biochem 80(1):79-87

Tezuka T, Tonomura K (1978) Purification and properties of a second enzyme catalyzing the splitting of carbon-mercury linkages from mercury-resistant Pseudomonas K-62. J Bacteriol 135(1):138-143

Tonomura K, Maeda K, Futai F, Nakagami T, Yamada M (1968) Stimulative vaporization of phenylmercuric acetate by mercury-resistant bacteria. Nature 217(5129):644-646

Uno Y, Kiyono M, Tezuka T, Pan-Hou H (1997) Phenylmercury transport mediated by merT-merP genes of Pseudomonas K-62 plasmid pMR26. Biol Pharm Bull 20(1):107-109

Vieira J, Messing J (1987) Production of single-stranded plasmid DNA. Methods Enzymol 153:3-11

Vuilleumier S, Chistoserdova L, Lee MC, Bringel F, Lajus A, Zhou Y, Gourion B, Barbe V, Chang J, Cruveiller S, Dossat C, Gillett W, Gruffaz C, Haugen E, Hourcade E, Levy R, Mangenot S, Muller E, Nadalig T, Pagni M, Penny C, Peyraud R, Robinson DG, Roche D, Rouy Z, Saenampechek C, Salvignol G, Vallenet D, Wu Z, Marx CJ, Vorholt JA, Olson MV, Kaul R, Weissenbach J, Medigue C, Lidstrom ME (2009) Methylobacterium genome sequences: a reference blueprint to investigate microbial metabolism of $\mathrm{C} 1$ compounds from natural and industrial sources. PLoS One 4(5):e5584. doi:10.1371/journal. pone.0005584.

doi:10.1186/2191-0855-3-41

Cite this article as: Sone et al:: Mercurial-resistance determinants in Pseudomonas strain K-62 plasmid pMR68. AMB Express 2013 3:41. 